\title{
Síndrome de Ekbom: revisão de literatura
}

\section{Ekbom's Syndrome: literature review}

Síndrome de Ekbom: revisión de la literatura

Izabely Lima Assunção ORCID: https://orcid.org/0000-0001-8262-9192

Universidade CEUMA, Brasil

E-mail: izabelyyyyy@gmail.com

Ana Karoline de Almeida Mendes ORCID: https://orcid.org/0000-0003-2654-7859 Universidade CEUMA, Brasi

E-mail: anakarolinemendesal@ hotmail.com

Kleber de Jesus Serrão Mendes Filho ORCID: https://orcid.org/0000-0003-2220-2589 Universidade CEUMA, Brasil

E-mail: Metodologiacientifica42@gmail.com

Pedro Cunha Mendes Neto

ORCID: https://orcid.org/0000-0003-4484-6828 Universidade CEUMA, Brasil

E-mail: Metodologiacientifica42@gmail.com

Paula Simplicio Viana de Carvalho ORCID: https://orcid.org/0000-0001-7509-111X Universidade CEUMA, Brasil E-mail: paulasimplicio@yahoo.com.br

Mariana Martins Calisto

ORCID: https://orcid.org/0000-0002-3040-9689 Universidade CEUMA, Brasil

E-mail: Metodologiacientifica42@gmail.com

Diego Barbosa Resende

ORCID: https://orcid.org/0000-0003-3429-7933

FTC Salvador, Brasil

E-mail: Metodologiacientifica42@gmail.com

Lara Vasconcelos Normando

ORCID: https://orcid.org/0000-0002-5649-9416 Universidade CEUMA, Brasil

E-mail: Metodologiacientifica42@gmail.com

Felipe de Queiroz Leite

ORCID: https://orcid.org/0000-0002-7422-4483 Universidade CEUMA, Brasil

E-mail: Metodologiacientifica42@gmail.com

Lise Feitosa Novais Miranda

ORCID: https://orcid.org/0000-0001-7804-2444 Universidade CEUMA, Brasil

E-mail: Metodologiacientifica42@gmail.com

Camila Costa Lacerda de Sousa ORCID: https://orcid.org/0000-0003-3597-713X Universidade CEUMA, Brasil

E-mail: Metodologiacientifica42@gmail.com

João Victor Carvalho da Paz

ORCID: https://orcid.org/0000-0002-2896-1079 Universidade CEUMA, Brasil

E-mail: Metodologiacientifica42@gmail.com

Ana Letícia Lopes Abreu Silva

ORCID: https://orcid.org/0000-0003-3210-5424 Universidade CEUMA, Brasil E-mail: uerbaleticia@gmail.com

Suzana Bastos Jácome de Souza ORCID: https://orcid.org/0000-0001-6530-4871 Instituto de Educação Superior do Vale do Parnaíba, Brasil

E-mail: suzanabastosjacome@bol.com.br 
Tarcízio Santos Murta Filho

ORCID: https://orcid.org/0000-0002-8963-3853 Universidade CEUMA, Brasil

E-mail: Tarciziomurtaf@outlook.com

Aléxia Mourão Alves Carvalhal

ORCID: https://orcid.org/0000-0003-4357-3602 Universidade CEUMA, Brasil

E-mail: alexia_mourao@hotmail.com

Karine Carneiro Fonseca

ORCID: https://orcid.org/0000-0003-3064-9778 Universidade CEUMA, Brasil E-mail: kfmedd@gmail.com

Renato Marcelo Silva Alexandre Filho

ORCID: https://orcid.org/0000-0002-7323-4276 Universidade CEUMA, Brasil

E-mail: Metodologiacientifica42@gmail.com

Isabela Teixeira dos Santos Silva

ORCID: https://orcid.org 0000-0002-3151-7331 Universidade CEUMA, Brasi

E-mail: isabelateixeira02@hotmail.com

Ilanna Cliscia Vieira Almeida

ORCID: https://orcid.org/0000-0001-9428-7273 Universidade CEUMA, Brasil

E-mail: cliscialmeida@hotmail.com

Rafael Pavão Gonçalves

ORCID: https://orcid.org/0000-0002-5234-3086 Universidade CEUMA, Brasi

E-mail: Metodologiacientifica42@gmail.com Mikael Coutinho Silva

ORCID: https://orcid.org/0000-0003-2243-9690 Universidade CEUMA, Brasil

E-mail: Metodologiacientifica42@gmail.com

Flávia Carneiro Pereira

ORCID: https://orcid.org/0000-0003-3650-8669 Universidade CEUMA, Brasil

E-mail: flaviacpraz@icloud.com

Suzana Bastos Jácome de Souza

ORCID: https://orcid.org/0000-0001-6530-4871 Instituto de Educação Superior do Vale do Parnaíba, Brasil

E-mail: suzanabastosjacome@bol.com.br

Camila Ribeiro Alvarez

ORCID: https://orcid.org/0000-0002-2706-6806

Universidade de Uberaba, Brasil

E-mail: camilaribalva@hotmail.com

Eloá Weba Costa

ORCID: https://orcid.org/0000-0003-2705-8205 Universidade CEUMA, Brasil

E-mail: eloa.weba.costa@gmail.com

Júlia de Souza Novais Mendes

ORCID: https://orcid.org/0000-0002-7605-4342 Universidade CEUMA, Brasil

E-mail: julia_mendes_23@hotmail.com

Juliana Oliveira Borges

ORCID: https://orcid.org/0000-0002-7605-4342

Universidade CEUMA, Brasil

E-mail: Metodologiacientifica42@gmail.com

Amanda Caroline dos Santos Matos

ORCID: https://orcid.org/0000-0003-0180-365X

Universidade CEUMA, Brasil

E-mail: Metodologiacientifica42@gmail.com

\section{Resumo}

A síndrome de Ekbom leva os pacientes a apresentarem uma ideia delirante e persistente de que vermes e micróbios os infestam. Este artigo se trata de uma revisão de literatura, na qual foram utilizadas as seguintes plataformas como base de dados para pesquisa: PubMed, Lilacs, Cochrane Library e Scientific Eletronic Library On-line (SciELO). Foram usados relatos de caso publicados no período entre 2017-2021, nas línguas portuguesa e inglesa, que abordavam as medicações utilizadas no tratamento da Síndrome de Ekbom. A análise bibliográfica foi realizada por meio da leitura criteriosa dos artigos baseados nos critérios de inclusão e exclusão citados. Dos 79 resultados obtidos, apenas 11 abordaram de forma clara e objetiva sobre o tema da pesquisa, sendo utilizados na confecção do artigo. Dessa forma, dentre os principais distúrbios psiquiátricos associados ao desenvolvimento da síndrome podem-se citar: 
depressão, esquizofrenia, e ansiedade, sendo que a demência por corpos de levy também foi relatada como possível desencadeadora da doença, ademais, a prevalência da Síndrome de Ekbom é maior em mulheres entre 50-60 anos. Com isso, tornou-se perceptível que a síndrome de Ekbom é uma doença de considerável prevalência na sociedade, e que devido à sua fisiopatologia desconhecida necessita de pesquisas mais aprofundadas sobre o assunto.

Palavras-chave: Síndrome de Ekbom; Ideia delirante; Transtorno psiquiátrico.

\begin{abstract}
Ekbom syndrome leads patients to have a persistent, delusional idea that worms and microbes infest it. This article is a systematic literature review, in which the following platforms were used as a database for the research: PubMed, Lilacs, Cochrane Library and Scientific Electronic Library On-line (SciELO). Case reports published in the period 2017-2021, in Portuguese and English, which addressed the medications used in the treatment of Ekbom syndrome, were used. A bibliographic analysis was carried out by carefully reading the articles based on the aforementioned inclusion and exclusion criteria. Of the 79 results obtained, only 11 addressed the research topic in a clear and objective manner, being used in the preparation of the article. Thus, the main psychiatric disorders associated with the development of the syndrome include: depression, schizophrenia, and anxiety, and dementia caused by levy bodies was also reported as a possible trigger of the disease, in addition, the prevalence of Ekbom's Syndrome it is higher in women aged 50-60 years. With this, it became noticeable that Ekbom syndrome is a disease predictive of prevalence in society, and that, due to its unknown pathophysiology, it needs further research on the subject.
\end{abstract}

Keywords: Ekbom's Syndrome; Delusional idea; Psychiatric disorder.

\title{
Resumen
}

El síndrome de Ekbom hace que los pacientes tengan una idea delirante y persistente de que los gusanos y los microbios lo infestan. Este artículo es una revisión de la literatura, en la que se utilizaron las siguientes plataformas como base de datos para la investigación: PubMed, Lilacs, Cochrane Library y Scientific Electronic Library On-line (SciELO). Se utilizaron informes de casos publicados en el período 2017-2021, en portugués e inglés, que abordaron los medicamentos utilizados en el tratamiento del síndrome de Ekbom. Se realizó un análisis bibliográfico mediante la lectura atenta de los artículos en base a los criterios de inclusión y exclusión mencionados anteriormente. De los 79 resultados obtenidos, solo 11 abordaron el tema de investigación de manera clara y objetiva, siendo utilizados en la elaboración del artículo. Así, los principales trastornos psiquiátricos asociados con el desarrollo del síndrome incluyen: depresión, esquizofrenia y ansiedad, y también se reportó la demencia causada por cuerpos de retención como posible desencadenante de la enfermedad, además, la prevalencia del Síndrome de Ekbom es mayor en mujeres de 50 a 60 años. Con esto, se hizo notar que el síndrome de Ekbom es una enfermedad predictiva de prevalencia en la sociedad, y que, debido a su fisiopatología desconocida, necesita más investigación sobre el tema.

Palabras clave: Síndrome de Ekbom; Idea delirante; Desordem psiquiátrico.

\section{Introdução}

A síndrome de Ekbom leva os pacientes a assumirem uma atitude automutilante, como: coçar, cortar ou, até mesmo, lesionar a pele. O objetivo do indivíduo que é acometido por essa síndrome, ao realizar, tais ações é eliminar parasitas, visto que esse transtorno tem relação com infestação parasitária e acarofobia - tipo de hipocondria em que o indivíduo apresenta um medo mórbido, caracterizado por medo irracional e compulsivo de animais microscópicos em contato com a pele (Goi \& Scharlau, 2007). Dessa forma, os pacientes apresentam uma ideia delirante e persistente de que vermes e micróbios os infestam (Amâncio et al., 2002).

A síndrome de Ekbom acomete principalmente mulheres na idade senil ou pré-senil, acometendo 1,9/100.000 por ano, mais especificamente na quinta e sexta década de vida, com importante impacto socioeconômico, tendo prevalência maior em mulheres que em homens (Andrade, 2017).

Assim, o protótipo de pacientes com delírio parasitário são mulheres com idade média de 58 anos, isoladas socialmente, divorciadas ou viúvas e com baixa escolaridade. Com isso, tais características são fatores de risco para o desenvolvimento do transtorno. Dessa forma, mulheres são mais capazes de induzir o transtorno que homens (Godoy \& Godoy, 2020).

O paciente acometido por essa síndrome sofre de psicose sintomáticas, com delírios e ou alucinações fixas e indubitáveis, de que está sendo infestado, parasitado ou dominado por parasitas e/ ou objetos inanimados (Zamora Rodríguez, F. J., 2011). 
A fisiopatologia da síndrome de Ekbom é desconhecida, havendo lesões de áreas importantes do cérebro e alterações na dopamina, possuindo relação com avitaminoses e diabetes mellitus (Alves, Martelli, Fogagnolo, \& Nassif, 2010). Há ainda teoria neurológicas no qual haveria lesões em certas áreas do encéfalo como substância subcortical, temporal, sistema límbico, região posteriores do hemisfério esquerdo; ou ainda lesões ou disfunções nas regiões do córtex frontal, tálamo, envolvidas na etiologia da síndrome. Estudos recentes demostram envolvimento do transportador de dopamina no corpo estriado (Muguet \& de Oliveira Nogueira).

Sobre o desenvolvimento da doença há também a teoria do envolvimento de desordens mentais somatiformes, no qual sintomas somáticos desagradáveis, que o paciente já está sentindo, são amplificados e perpetuados quando o mesmo tem contato com outros indivíduos infectados, através de recursos midiáticos ou agentes de saúde pública quando noticiam sobre o assunto (Bruno et al., 1996).

Sobre sinais e sintomas da doença, o paciente relata histórico de idas ao clínico geral e até dermatologistas, e permanência da sintomatologia do paciente mesmo com interferência de medicações. Há também histórico de uso de medicações anti- prurido, anti-verme, anti-parasitas, anti-fungicas. O paciente também pode atribuir o desenvolvimento de sua patologia aos seus animais de estimação, indo várias vezes ao veterinário, tratando o animal como doente (Alves et al., 2010).

O padrão de vida é comprometido com higienização exagerada tanto do paciente como do ambiente em que ele convive na tentativa de alimentar algum patógeno. Lesões dermatologistas devido a alucinações táteis com ou sem prurido fazem parte de sintomatologia frequente em paciente que apresentam síndrome de Ekbom (BRUNO et al., 1996).

O diagnóstico é de exclusão e sua forma primária ainda é excludente, devendo o médico realizar anamnese detalhada, uso de drogas lícitas ou ilícitas, avitaminoses por dietas inadequadas, condições de higiene pessoal (González, L., 2010).

Os critérios presentes no DSM-5 relatam de presença de delírio por duração de 1 mês, descarte de esquizofrenia e alucinações específicas ao contexto de infestação e parasitose. A funcionalidade do paciente não é totalmente comprometida e seu comportamento não é bizarro ou estranho. Se o indivíduo tiver diagnóstico de mania ou depressão, tais doenças ocorrem em menor tempo que no delírio, e a doença não pode ser atribuída a qualquer causa médica, relacionada a uso de quaisquer substâncias ou mais bem explicada por outra doença mental (Munoz, H., \& Bayona, 2015).

$\mathrm{O}$ tratamento envolve soma de empenhos entre dermatologista e psiquiatras. As indicações de tratamento envolvem risco de suicídio e risco para outras pessoas. Sobre tratamento medicamentoso, as primeiras gerações de antipsicóticos não são utilizadas por questões de efeitos adversos como alterações posturais, extrapiramidais e risco de internação. Logo são usados de segunda linha, como risperidona (Andrade, 2017; Goi \& Scharlau, 2007).

Tendo em vista que a síndrome de Ekbom é um transtorno psiquiátrico que necessita de atenção, pois apenas com diagnósticos e tratamentos corretos há prognóstico, o objetivo do trabalho foi realizar uma revisão de literatura associando a síndrome de Ekbom, sinais e sintomas clínicos, e tratamento.

\section{Metodologia}

Este artigo se trata de uma revisão de literatura, na qual foram utilizadas as seguintes plataformas como base de dados para pesquisa: PubMed, Lilacs, Cochrane Library e Scientific Eletronic Library On-line (SciELO). Foram usados relatos de caso publicados no período entre 2017-2021, nas línguas portuguesa e inglesa, que abordavam a sintomatologia e tratamento utilizado na Síndrome de Ekbom.

Os descritores utilizados nesta pesquisa estão inclusos no Medical Subject Headings (MeSH) e no DeCs (Descritores em Saúde), nos idiomas português e inglês respectivamente: Delírio de Parasitose (Delusional Parasitosis) OR Delírio de Parasitose de Ekbom (Ekbom Delusional Parasitosis). 
Nesta revisão de literatura, os critérios de exclusão usados foram os seguintes: resumos em eventos, editoriais, artigos duplicados, artigos sobre animais e artigos que não cumpriam os critérios de inclusão.

\section{Resultados e Discussão}

A análise bibliográfica foi realizada por meio da leitura criteriosa dos artigos baseados nos critérios de inclusão e exclusão citados. Dos 79 resultados obtidos, apenas 11 abordaram de forma clara objetiva sobre o tema da pesquisa, sendo utilizados na confecção do artigo. Todos as literaturas selecionadas serão expostas no Quadro 1.

Quadro 1. Artigos selecionados para pesquisa.

\begin{tabular}{|c|c|c|c|}
\hline Autor, Ano & Título & Caso & Tratamento \\
\hline $\begin{array}{l}\text { (Tan, Zhang, Feng, \& } \\
\text { Liu, 2020) }\end{array}$ & $\begin{array}{l}\text { A patient with delusional parasitosis } \\
\text { secondary to herpes zoster and } \\
\text { comorbid depression: a case report }\end{array}$ & $\begin{array}{l}\text { - Mulher, } 69 \text { anos, com diagnóstico de } \\
\text { DP refratária secundária a herpes zoster } \\
\text { e depressão comórbida. }\end{array}$ & Fluvoxamina e Olanzapina. \\
\hline $\begin{array}{l}\text { (Mendonça, Teixeira, } \\
\text { \& Marinho, 2020) }\end{array}$ & $\begin{array}{l}\text { Ekbom Syndrome associated with } \\
\text { Lewy Body Dementia: A case } \\
\text { report }\end{array}$ & $\begin{array}{l}\text { - Homem, } 72 \text { anos, com início dos } \\
\text { sintomas aos } 70 \text { anos. Paciente com } \\
\text { diagnóstico de demência com corpos de } \\
\text { Lewy. }\end{array}$ & Citalopram e Rivastigmina \\
\hline $\begin{array}{l}\text { (Soltan-Alinejad, } \\
\text { Vahedi, Turki, \& } \\
\text { Soltani, 2021) }\end{array}$ & $\begin{array}{l}\text { A comprehensive entomological } \\
\text { survey and evaluation of the } \\
\text { efficacy of different therapies on a } \\
\text { suspected delusional parasitosis } \\
\text { case }\end{array}$ & $\begin{array}{l}\text { - Homem, } 40 \text { anos, histórico } \mathrm{de} \\
\text { Depressão. }\end{array}$ & $\begin{array}{lllll}\text { Risperidona } & (2-6 \mathrm{mg} / \mathrm{d}) & \mathrm{e} \\
\text { olanzapina } & (5 & \mathrm{mg} & / & \mathrm{d}) ; \\
\text { hipnotismo. } & & & \end{array}$ \\
\hline (Das \& Bagla, 2020) & $\begin{array}{l}\text { A rare case of co-existence of } \\
\text { delusional parasitosis, delusional } \\
\text { parasitosis by proxy and folie à } \\
\text { deux }\end{array}$ & $\begin{array}{l}\text { - Mulher, } 55 \text { anos, mãe da paciente } \\
\text { compartilhava da mesma crença. } \\
\text { - Mulher, } 37 \text { anos, sogra acreditou na } \\
\text { crença da paciente. } \\
\text { - Homem , } 67 \text { anos, esposa acreditava } \\
\text { firmemente na crença do paciente. }\end{array}$ & $\begin{array}{l}\text { Risperidona }(2-6 \mathrm{mg} / \mathrm{dia}) \text { por } 3 \\
\text { meses. }\end{array}$ \\
\hline $\begin{array}{l}\text { (Roulet \& Zdanowicz, } \\
\text { 2017) }\end{array}$ & $\begin{array}{l}\text { Delusional parasitosis treated by } \\
\text { atypical antipsychotic and selective } \\
\text { serotonin reuptake inhibitor: a case } \\
\text { report }\end{array}$ & $\begin{array}{l}\text { - Mulher, com início dos sintomas de } \\
\text { DP após interrupção do uso de } \\
\text { sertralina. }\end{array}$ & $\begin{array}{l}200 \mathrm{mg} \text { de Setralina e } 2 \mathrm{mg} \text { de } \\
\text { risperidona. }\end{array}$ \\
\hline $\begin{array}{lr}\text { Dipp } & \text { Vargas, } \\
\text { Salguedo } & \text { Siles, } \\
\text { Mendoza } & \text { López } \\
\text { Videla, \& } & \text { Burgoa } \\
\text { Seaone, 2020) } & \\
\end{array}$ & $\begin{array}{l}\text { Parasitose Dilusional Ou Síndrome } \\
\text { Ekbom }\end{array}$ & $\begin{array}{l}\text { - Homem, } 54 \text { anos, com diagnóstico de } \\
\text { esquizofrenia residual. }\end{array}$ & $\begin{array}{l}\text { Risperidona } 6 \mathrm{mg} / \mathrm{dia} \text {, por } 2 \\
\text { anos. }\end{array}$ \\
\hline $\begin{array}{lr}\text { (García } & \text { Tercero, } \\
\text { Landaluce } & \text { Pelligra, } \\
\text { Alonso Seco, \& Porta } \\
\text { González, 2020) }\end{array}$ & $\begin{array}{l}\text { Ekbom's syndrome: case report of } \\
\text { an older patient }\end{array}$ & $\mathrm{m}, 88$ anos. & 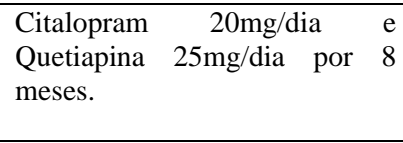 \\
\hline $\begin{array}{l}\text { Estrada, } \\
\text { Sharma, \& } \begin{array}{l}\text { Persaud- } \\
\text { 2019) }\end{array}\end{array}$ & $\begin{array}{l}\text { Organic etiology of delusional } \\
\text { parasitosis in the Hispanic } \\
\text { population: a case report }\end{array}$ & 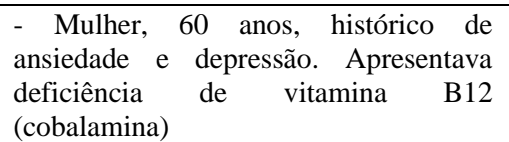 & $\begin{array}{l}\text { Paciente recebeu } 4 \text { doses } \\
\text { intramusculares de cobalamina, } \\
\text { com sintomas completamente } \\
\text { resolvidos após } 1 \text { mês. }\end{array}$ \\
\hline $\begin{array}{l}\text { (Whitfield, Krasniak, \& } \\
\text { Nguyen, 2021) }\end{array}$ & $\begin{array}{l}\text { Concurrent Delusions of Ocular } \\
\text { Parasitosis and Complex Visual } \\
\text { Hallucinations from Charles Bonnet } \\
\text { Syndrome Treated Successfully } \\
\text { with Aripiprazole in an Elderly } \\
\text { Male: A Case Report }\end{array}$ & - Homem, 78 anos. & Aripiprazol 6-15mg/dia. \\
\hline $\begin{array}{l}\text { (McKinnon \& Dow, } \\
2020)\end{array}$ & $\begin{array}{l}\text { Brief cognitive behavioural therapy } \\
\text { for post-stroke 'delusional } \\
\text { infestation' in a 71-year-old man: a } \\
\text { single case experimental design }\end{array}$ & $\begin{array}{l}\text { - Homem, } 70 \text { anos, com intolerância a } \\
\text { medicações antipsicóticas. }\end{array}$ & $\begin{array}{lr}\text { Paciente utilizou } & \text { sessões de } \\
\text { Terapia } & \text { Cognitiva } \\
\text { Comportamental. } & \end{array}$ \\
\hline (Jamaluddin, 2018) & $\begin{array}{l}\text { Delusional infestation successfully } \\
\text { controlled with typical } \\
\text { antipsychotic depot injection: An } \\
\text { illustrative case report. }\end{array}$ & $\begin{array}{l}\text { - Homem, } 36 \text { anos, com diagnóstico } \\
\text { prévio de esquizofrenia. }\end{array}$ & $\begin{array}{lcr}\text { Olanzapina } & 10-15 & \mathrm{mg} / \text { dia e } 7 \\
\text { sessões de derapias } & \text { de } \\
\text { eletroconvulsivas. A medicação } \\
\text { oral era difícil de monitorar, } \\
\text { logo foi realizada injeção de } \\
\text { depósito de decanoato de } \\
\text { flufenazina } 25-50 \mathrm{mg} / \mathrm{mês} \text {. }\end{array}$ \\
\hline
\end{tabular}

Fonte: Autores. 
Entre os artigos analisados, pôde-se perceber que, apesar de Andrade (2017) ter descrito na literatura que a prevalência da Síndrome de Ekbom seja maior em mulheres entre 50-60 anos, há mais relatos de caso descrevendo a terapia farmacológica em pacientes do sexo masculino. Nos casos selecionados, os pacientes com DP abaixo dessa faixa etária relataram ter histórico psiquiátrico prévio (Jamaluddin, 2018; Soltan-Alinejad et al., 2021).

Dentre os principais distúrbios psiquiátricos associados ao desenvolvimento da síndrome podem-se citar: depressão, esquizofrenia, e ansiedade, sendo que a demência por corpos de levy também foi relatada como possível desencadeadora da doença (Mendonça et al., 2020). Segundo Alvez, Martelli, Fogagnolo e Nassif (2010), a fisiopatologia da síndrome ainda não é totalmente conhecida, podendo estar relacionada a diversos fatores, especialmente doenças orgânicas e lesões em determinadas áreas do cérebro.

Em relação ao caso relacionado à deficiência de vitamina B12, é importante enfatizar que a DP pode se apresentar secundária a doenças orgânicas, como demência, anemia severa, hipovitaminose, diabetes, intoxicações, infecção por herpes zoster, dentre outras (Alves et al., 2010). Dessa forma, é de fundamental importância que seja realizada uma avaliação laboratorial completa para afastar possíveis diagnósticos diferenciais, visto que, como no caso relatado, o tratamento visa primeiramente tratar a hipovitaminose, uma vez que pode ser a causa do desenvolvimento dos sintomas de delírio e alucinatórios com insetos (Estrada et al., 2019).

No caso descrito por Tan, Zhang, Feng e Liu (2020), uma paciente com herpes zoster abdominal não tratada adequadamente associou o prurido característico da herpes que relatava há três anos, a pequenos insetos que acreditava estarem rastejando em sua pele. Como a síndrome de ekbom está relacionada a delírios e/ou alucinações de que está sendo parasitado, o caso relatado pode estar associado à ansiedade e tensão mental causada pelo prurido por um grande período de tempo se melhora, contribuindo para a progressão da doença.

Em relação à terapia farmacológica, observou-se nas obras selecionadas que em essência o ideal a ser utilizado para tratar os sintomas positivos da psicose presente na síndrome são os antipsicóticos, em especial a risperidona e olanzapina (Das \& Bagla, 2020; Dipp Vargas et al., 2020; Roulet \& Zdanowicz, 2017; Soltan-Alinejad et al., 2021; Tan et al., 2020). Nesses casos, com o intuito de tratar os delírios e alucinações com o menor efeito adverso possível, é recomendado o uso dos antipsicóticos de segunda geração já citados, visto que alterações posturais e extrapiramidais são mais prevalentes quando se opta pelos de primeira geração (Andrade, 2017; Goi \& Scharlau, 2007).

Além dos antipsicóticos, pode-se associar ao tratamento os antidepressivos da classe dos inibidores seletivos de receptação de serotonina, como a fluvoxamina, sertralina e citalopram (Mendonça et al., 2020; Roulet \& Zdanowicz, 2017; Tan et al., 2020). Esses antidepressivos são utilizados especialmente devido ao estado de fobia obsessiva em que o paciente se encontra, visto que acredita estar sendo infestado por parasitas na pele.

Nos casos que apresentam diagnóstico concomitante com esquizofrenia de forma grave, é interessante analisar se o paciente adere ao uso de medicações orais ou se é necessário que seja realizado injeção de depósito da medicação para melhora do paciente, como o deconoato de flufenazina, que foi prescrita no caso relatado por Jamaluddin (2018).

Além da terapia farmacológica é essencial analisar a importância da psicologia no tratamento da síndrome de Ekbom. No caso relatado por McKinnon e Dow (2020), por exemplo, o paciente apresentava intolerância a medicações antipsicóticas, que são de fundamental importância para a melhora do quadro clínico do paciente, sendo assim, optou-se por seguir o tratamento apenas com sessões de Terapia Cognitivo-Comportamental (TCC). Nesse caso, o paciente obteve uma melhora clínica considerável na área de angústia e ansiedade, no entanto ainda possuía a crença de que a infestação era real, se mantendo necessário o seguimento na terapia por três meses. 


\section{Conclusão}

De acordo com as literaturas analisadas, tornou-se perceptível que a síndrome de Ekbom é uma doença de considerável prevalência na sociedade, e que devido à sua fisiopatologia desconhecida necessita de pesquisas mais aprofundadas sobre o assunto, especialmente em casos secundários a desordens orgânicas, uma vez que é necessário saber a causa primária para um tratamento eficaz.

Por ter como característica principal o prurido associado a delírios e alucinações, os profissionais mais capacitados a tratar dessa doença são dermatologistas e psiquiatras, uma vez que é necessária uma avaliação laboratorial completa antes do diagnóstico conclusivo. Assim, é de fundamental importância que esses profissionais conheçam o tratamento e auxiliem o paciente da melhor forma possível em sua adesão, visto que, por conta dos sintomas psicóticos positivos, há a crença real de que há parasitas em sua pele constantemente.

\section{Referências}

Alves, C. J. M., Martelli, A. C. C., Fogagnolo, L., \& Nassif, P. W. (2010). Síndrome de Ekbom secundária a transtorno orgânico: relato de três casos. Anais Brasileiros de Dermatologia, 85, 541-544.

Amâncio, E. J., Peluso, C. M., Santos, A. C. G., Magalhães, C. C. P., Pires, M. F. C., Peña Dias, A. P., \& Debs, F. A. A. (2002). Síndrome de Ekbom e torcicolo espasmódico: relato de caso. Arquivos de neuro-psiquiatria, 60, 155-158.

Andrade, L. P. (2017). Síndrome de Ekbom: um relato de caso.

Bruno, C. A. F., Castelo-Branco, A. L., \& Lopez, J. (1996). Sindrome de Ekbom e disturbio delirante do tipo somatico: revisao do conceito e relato de um caso clinico. Informacao Psiquiatrica, 94-97.

Das, A., \& Bagla, P. (2020). A rare case of co-existence of delusional parasitosis, delusional parasitosis by proxy and folie à deux. Schizophr Res, 220, 269270. doi:10.1016/j.schres.2020.04.005

Dipp Vargas, C., Salguedo Siles, S. F., Mendoza López Videla, J. N., \& Burgoa Seaone, M. (2020). Delirio de parasitosis o síndrome de Ekbom. Revista Médica La Paz, 26, 46-49.

Estrada, E., Persaud-Sharma, D., \& Corredor, R. G. (2019). Organic etiology of delusional parasitosis in the Hispanic population: a case report. Archives of Clinical Psychiatry (São Paulo), 46, 51-51.

García Tercero, E., Landaluce Pelligra, V., Alonso Seco, M., \& Porta González, L. A. (2020). Síndrome de Ekbom: reporte de un caso en un paciente anciano. Revista Clínica de Medicina de Familia, 13, 81-84.

Godoy, A. U., \& Godoy, B. U. (2020). Síndrome de Ekbom secundária a hipotireoidismo. Revista de APS, 23.

Goi, P. D., \& Scharlau, C. T. (2007). Síndrome de Ekbom acompanhada de automutilação. Revista de Psiquiatria do Rio Grande do Sul, $29(1), 97-99$.

González, L., Torales, J., Arce, A., Di Martino, B., Valdovinos, G., Rodríguez, M., ... \& Bolla, L. (2010). Síndrome de Ekbom. A propósito de un caso [Ekbom's syndrome. A case report]. Art Terap Dermatol, 33, 140-145.

Jamaluddin, R. (2018). Delusional infestation successfully controlled with typical antipsychotic depot injection: An illustrative case report. Asian journal of psychiatry, 31, 69-70.

McKinnon, A. I., \& Dow, R. (2020). Brief cognitive behavioural therapy for post-stroke 'delusional infestation'in a 71-year-old man: a single case experimental design. Behavioural and Cognitive Psychotherapy, 48(6), 717-724.

Mendonça, F. J. P. d., Teixeira, I. A., \& Marinho, V. (2020). Ekbom syndrome associated with Lewy body dementia: a case report. Dementia \& neuropsychologia, 14, 83-87.

Muguet, B. O., \& de Oliveira Nogueira, G. Q. RElato de caso: síndrome de Ekbom um desafio diagnóstico e terapêutico.

Munoz, H., \& Bayona, L. (2015). Síndrome de Ekbom: a propósito de un caso. Revista colombiana de psiquiatría, 44(1), 61-65.

Roulet, T., \& Zdanowicz, N. (2017). Delusional parasitosis treated by atypical antipsychotic and selective serotonin reuptake inhibitor: a case report. Psychiatria Danubina, 29(Suppl 3), 219-221.

Soltan-Alinejad, P., Vahedi, M., Turki, H., \& Soltani, A. (2021). A comprehensive entomological survey and evaluation of the efficacy of different therapies on a suspected delusional parasitosis case. Brain and Behavior, 11(1), e01945.

Tan, G., Zhang, H., Feng, W., \& Liu, Z. (2020). A patient with delusional parasitosis secondary to herpes zoster and comorbid depression: a case report. Psychogeriatrics: the Official Journal of the Japanese Psychogeriatric Society. 
Research, Society and Development, v. 10, n. 16, e302101623957, 2021

(CC BY 4.0) | ISSN 2525-3409 | DOI: http://dx.doi.org/10.33448/rsd-v10i16.23957

Whitfield, N. T., Krasniak, A. E., \& Nguyen, H. T. (2021). Concurrent Delusions of Ocular Parasitosis and Complex Visual Hallucinations from Charles Bonnet Syndrome Treated Successfully with Aripiprazole in an Elderly Male: A Case Report. Permanente Journal, 25(1).

Zamora Rodríguez, F. J., Benítez Vega, C., Calderón López, P., Guisado Macías, J. A., Teva García, M. I., \& Sánchez-Waisen Hernández, M. R. (2011). Un caso de síndrome de Ekbom. Psiquiatr. biol.(Internet), 42-44. 